\title{
THE GENUS FIELD AND GENUS NUMBER IN ALGEBRAIC NUMBER FIELDS
}

\author{
YOSHIOMI FURUTA ${ }^{1,}$
}

\section{Dedicated Professor KIYoshi Noshiro on his 60th birthday}

Let $k$ be an algebraic number field and $K$ be its normal extension of finite degree. Then the genus field $K^{*}$ of $K$ over $k$ is defined as the maximal unramified extension of $K$ which is obtained from $K$ by composing an abelian extension over $k^{2}$. We call the degree $\left(K^{*}: K\right)$ the genus number of $K$ over $k$.

In the case where $k$ is the rational number field, the genus number is studied by Hasse [2] for quadratic extensions, by Iyanaga and Tamagawa [3] and by Leopoldt [6] for abelian extensions, and by Fröhlich [1], [1'] for normal extensions.

At the present time, there is no difficulty to treat the genus number in general, for which, however, no convenient literature is available. So, in this rather expository paper, we shall give a general formula for the genus number, which would have some meaning especially in the investigation of the class number relation ${ }^{3 j}$.

1. For any finite or infinite prime $\mathfrak{p}$ of $k$ we denote by $k_{\mathfrak{p}}$ the $\mathfrak{p}$-completion of $k ; U_{\mathfrak{p}}$ the unit group of $k_{\mathfrak{p}} ; J_{k}$ the idele group of $k$, into which we embed $k^{\times}$and $k_{\mathfrak{p}}^{\times}$in usual way ${ }^{4}$; and $U_{k}=\prod_{\mathfrak{p}} U_{\mathfrak{p}}$ the unit idele group of $k$.

A subgroup $H$ of $J_{k}$ is called admissible if $H$ is a closed subgroup of finite index in $J_{k}$ and contains $k^{\times}$. Then an admissible subgroup of $J_{k}$ and an abelian extension over $k$ of finite degree correspond to each other by the class field theory.

For an Galois extension $K / k$ we denote by $N_{K / k}$ the norm from $K$ to $k$ and

Received June 17, 1966.

1) The author wishes to express his hearty thanks to Professor T. Kubota and H. Yokoi for their valuable advice.

2) For normal extensions $K / k$ this definition is according to Froblich [1].

3) Cf. Fröhlich [1], Iwasawa [4], Kuroda [5] and Yokoyama [7].

1) We mean by $k \times$ the multiplicative group of all non-zero elements of $k$. 
we will often omit the suffix when its meaning is obvious.

Lemma 1. Let $K$ be an extension over $k$ of finite degree, $\hat{H}$ be an admissible subgroup of $J_{K}$ and $\hat{K}$ be the class field over $K$ corresponding to $\hat{H}$. Let further $\hat{K}_{0}$ be the maximal abelian subfield of $\hat{K}$ over $k . \quad$ Then $k^{\times}\left(N_{K / k} \hat{H}\right)$ is the admissible subgroup of $J_{k}$ corresponding to $\hat{K}_{0}$.

Proof. Denote by $\hat{H}_{0}$ resp. $H_{0}$ be the admissible subgroup of $J_{K}$ resp. $J_{k}$ corresponding to $K \hat{K}_{0}$ resp. $\hat{K}_{0}$. Then the translation theorem of the class field theory implies that $\hat{H}_{0}$ is generated by $K^{\times}$and by all a of $J_{K}$ such that $N_{K / k} a \in H_{0}$. Hence $H_{0} \supset k^{\times} \cdot N\left(K^{\times} \hat{H}_{0}\right)=k^{\times} \cdot N \hat{H}_{0} \supset k^{\times} \cdot N \hat{H}$, because $K \hat{K}_{0} \subset \hat{K}$ implies $\hat{H}_{0} \supset \hat{H}$. On the other hand $\left(U_{\mathfrak{p}}: N U_{\mathfrak{B}}\right)$ is finite and moreover equal to 1 for almost all $\mathfrak{p}$. This implies that $N \hat{H} \cap U_{k}$ is an open subgroup of $J_{k}$ and hence $k^{\times} \cdot N \hat{H}$ is an admissible subgroup of $J_{k}$. Let $K_{1}$ be the class field over $k$ corresponding to $k^{\times} \cdot N \hat{H}$, and let $\hat{H}_{1}$ be the admissible subgroup of $J_{K}$ corresponding to $K K_{1}$. Then the translation theorem of the class field theory implies that $\hat{H}_{1}$ is generated by $K^{\times}$and by all $a \in J_{K}$ such that $N a \in k^{\times} \cdot N \hat{H}$. Hence $\hat{H}_{1} \supset \hat{H}$, which implies $K K_{1} \subset \hat{K}$. Moreover we have $K_{1} \subset \hat{K}_{0}$, because $K_{1}$ is abelian over $k$. Thus we have $k^{\times} \cdot N \hat{H} \supset H_{0}$ and the lemma is proved.

2. Let $K$ be as before a normal extension over $k$ of finite degree and $K^{*}$ be the genus field of $K$ over $k$, which is defined as the maximal unramified extension of $K$ obtained by composing an abelian extension over $k$. Now denote by $K_{0}^{*}$ the maximal abelian subfied of $K^{*}$ over $k$. Then $K^{*}$ is the composite of $K$ and $K_{0}^{*}$. Let $A_{K}$ be the Hilbert class field of $K$, that is, the maximal unramified abelian extension over $K$. Then obviousely $K_{0}^{*}$ is the maximal abelian subfield of $A_{K}$ over $k$. Since $K^{\times} U_{K}$ is the admissible subgroup of $J_{K}$ corresponding to $A_{K}$, lemma 1 implies the following

Proposition 1. Notations being as above, let further $H^{*}$ be the admissible subgroup of $J_{k}$ corresponding to $K_{0}^{*}$. Then we have

$$
H^{*}=k^{\times} \prod_{\mathfrak{p}} N U_{\mathfrak{B}} \text {, }
$$

where the product is taken over all finite or infinite primes $\mathfrak{p}$, and for each $\mathfrak{p}, \mathfrak{P}$ is any one of primes of $K$ dividing $\mathfrak{p}$.

3. If especially $K$ is abelian over $k$, then its genus field $K^{*}$ is also abelian, and we have $K_{0}^{*}=K^{*}$. Moreover $H^{*}$ is expressed by means of the admissible. 
subgroup $H$ of $J_{k}$ corresponding to $K$ as in the following proposition, although this is not necessary for the theorem of this paper.

Proposition 2. Let $K$ be an abelian extension of $k$ and $H$ be the corresponding admissible subgroup of $J_{k}$. Let further $H^{*}$ be the admissible subgroup of $J_{k}$ corresponding to the genus field of $K$ over $k$. Then we have

$$
H^{*}=k^{\times} \prod_{\mathfrak{p}}\left(H \cap U_{\mathfrak{p}}\right),
$$

where the product is taken over all finite and infinite primes of $k$.

Proof. Let $u_{\mathfrak{p}} \in U_{\mathfrak{p}}$, where $U_{\mathfrak{p}}$ is embeded in $J_{k}$, then since the global norm residue symbol is the product of local ones, we have $\left(u_{\mathfrak{p}}, K / k\right)=\left(u_{\mathfrak{p}}, K_{\mathfrak{F}} / k_{\mathfrak{p}}\right)$. Hence $H \cap U_{\mathfrak{p}}$ consists of all $u_{\mathfrak{p}} \in U_{\mathfrak{p}}$ such that $\left(u_{\mathfrak{p}}, K_{\mathfrak{P}} / k_{\mathfrak{p}}\right)=1$, and this implies $H \cap U_{\mathfrak{p}}=N K_{\mathfrak{B}} \cap U_{\mathfrak{p}}=N U_{\mathfrak{B}}$. Since $N U_{K}=\prod_{\mathfrak{p}} N U_{\mathfrak{B}}$, the proposition follows easily from proposition 1 .

4. Remark. In the case where $k$ is the rational number field $Q$ and $K$ is abelian over $Q$, Leopoldt [6] showed that the congruent ideal character group corresponding to the genus field $K^{*} / Q$ is generated by "Auflösung" of the congruent ideal character group corresponding to $K / Q$. In this case we have $J_{Q}=Q^{\times} U$. Hence the idele character group corresponding to $K^{*} / Q$ is determined as the character group of $U / \Pi_{\mathfrak{p}}\left(H \cap U_{\mathfrak{p}}\right) \bmod Q^{\times}$, and this is generated by characters of $U /\left(H \cap U_{\mathfrak{p}}\right) \prod_{\mathfrak{q} \neq \mathfrak{p}} U_{\mathfrak{q}} \bmod Q^{\times}$, where $\mathfrak{p}$ runs over all primes of $k$. We can show easily that the congruent ideal character group corresponding to this idele character group is exactly the above "Auflösung".

5. Let again $K$ be a normal extension of $k$ of finite degree, and $K^{*}$ be its genus field. In order to estimate the genus number $\left(K^{*}: K\right)$ we first prove the following

Lемма 2. Let $K$ be a normal extension of $k$ of finite degree, $\mathfrak{p}$ be a finite or infinite prime of $k$, and $\mathfrak{P}$ be a prime of $K$ dividing $\mathfrak{p}$. Then the index $\left(U_{\mathfrak{p}}: N U_{\mathfrak{B}}\right)$ is equal to the ramification index of the maximal abelian subfield of $K_{\uparrow}$ over $k_{p}$.

Proof. Let $K_{\mathfrak{\beta}}^{\prime}$ be the maximal abelian subfield of $K_{\mathfrak{B}}$ over $k_{\mathfrak{p}}$. Then we have $N K_{\mathfrak{B}}=N K_{\mathfrak{B}}^{\prime}$ by the local class field theory. Hence $\left(U_{\mathfrak{p}}: N U_{\mathfrak{B}}\right)=$ $\left(U_{\mathfrak{p}}: U_{\mathfrak{p}} \cap N K_{\mathfrak{p}}\right)=\left(U_{\mathfrak{p}}: U_{\mathfrak{p}} \cap N K_{\mathfrak{p}}^{\prime}\right)=\left(U_{\mathfrak{p}} \cdot N K_{\mathfrak{p}}^{\prime}: N K_{\mathfrak{p}}^{\prime}\right)$. On the other hand $U_{\mathfrak{p}} \cdot N K_{\mathfrak{B}}^{\prime}$ is the subgroup of $k_{\mathfrak{p}}$ corresponding to the inertia field of $K_{\mathfrak{p}}^{\prime}$ over 
$k_{\mathfrak{p}}$ and $N K_{\mathfrak{\beta}}^{\prime}$ is that of to $K_{\mathfrak{\beta}}^{\prime}$ over $k_{\mathfrak{p}}$ by means of the local class field theory. Hence the last index is equal to the ramification index of $K_{\beta}^{\prime}$ over $k_{p}$, which is to be proved.

Notations being as above let further $K_{0}^{*}$ be the maximal abelian subfield of $K^{*}$ over $k$ and $H^{*}$ be the admissible subgroup of $J_{k}$ corresponding to $K_{0}^{*}$. Denote by $\varepsilon$ any unit of $k$, and by $\eta$ a unit of $k$ which is everywhere locally norm, that is, for each prime $\mathfrak{P}$ of $K$ there exists an element $\alpha_{\mathfrak{\beta}}$ of $K_{\mathfrak{B}}$ such that $\eta=N \alpha_{\mathfrak{B}}$. Then we have

Theorem. The genus number of a normal extension $K$ over $k$ is equal to

$$
\frac{h_{k} \underset{\mathfrak{p}}{\prod_{\mathfrak{p}}} e^{\prime}}{\left(K_{0}: k\right)(\varepsilon: \eta)}
$$

where $h_{k}$ is the class number of $k, e_{p}^{\prime}$ is the ramification index of the maximal abelian subfield $K_{\mathfrak{p}}$ over $k_{\mathfrak{p}}, K_{0}$ is the maximal abelian subfield of $K$ over $k$, and $\mathfrak{p}$ runs over all finite and infinite primes of $k$.

Proof. Since $K K_{0}^{*}=K^{*}$ and $K \cap K_{0}^{*}=K_{0}$, the genus number $\left(K^{*}: K\right)$ is equal to $\left(K_{0}^{*}: K_{0}\right)$. We have

$$
\left(K_{0}^{*}: K_{0}\right)=\frac{\left(K_{0}^{*}: k\right)}{\left(K_{0}: k\right)}=\frac{\left(J_{k}: H^{*}\right)}{\left(K_{0}: k\right)}=\frac{\left(J_{k}: k^{*} U\right)\left(k^{\times} U: H^{*}\right)}{\left(K_{3}: k\right)}=\frac{h_{k}\left(k^{\times} U: H^{*}\right)}{\left(K_{0}: k\right)} .
$$

Since $H^{*}=k^{\times} \cdot \prod_{\mathfrak{p}} N U_{\mathfrak{P}}$ by proposition 1 , we have moreover

$$
\left(k^{\times} U: H^{*}\right)=\left(H^{*} U: H^{*}\right)=\left(U: H^{*} \cap U\right)=\frac{\left(U: \prod_{\mathfrak{p}} N U_{\mathfrak{B}}\right)}{\left(H^{*} \cap U: \prod_{\mathfrak{p}} N U_{\mathfrak{B}}\right)},
$$

and by lemma 2

$$
\left(U: \prod_{\mathfrak{p}} N U_{\mathfrak{P}}\right)=\prod_{\mathfrak{p}}\left(U_{\mathfrak{p}}: N U_{\mathfrak{P}}\right)=\prod_{\mathfrak{p}} e_{\mathfrak{p}}^{\prime}
$$

Hence in order to prove the theorem it remains only to show ( $H^{*} \cap U$ : $\left.\prod_{\mathfrak{p}} N U_{\mathfrak{B}}\right)=(\varepsilon: \eta)$. Obviousely $H^{*} \cap U=k^{\times} \cdot \prod_{\mathfrak{p}} N U_{\mathfrak{\beta}} \cap U \supset\left(k^{\times} \cap U\right) \cdot \prod_{\mathfrak{p}} N U_{\mathfrak{\beta}}$. Conversely let $\alpha u \in k^{\times} \cdot \prod_{\mathfrak{p}} N U_{\mathfrak{\beta}} \cap U, \alpha \in k^{\times}, u \in \prod_{\mathfrak{p}} N U_{\mathfrak{B}}$, then we have $\alpha \in u^{-1} U \subset U$, and $\alpha \in k^{\times} \cap U$. Hence $H^{*} \cap U=\left(k^{\times} \cap U\right) \cdot \prod_{\mathfrak{p}} N U_{\mathfrak{B}}$ and we see $\left(H^{*} \cap U: \prod_{\mathfrak{p}} N U_{\mathfrak{B}}\right)$ $=\left(\left(k^{\times} \cap U\right) \cdot \prod_{\mathfrak{p}} N U_{\mathfrak{B}}: \prod_{\mathfrak{p}} N U_{\mathfrak{B}}\right)=\left(k^{\times} \cap \stackrel{\mathfrak{p}}{U}:\left(k^{\times} \cap U\right) \cap \prod_{\mathfrak{p}} N U_{\mathfrak{B}}\right)=\left(k^{\times} \cap U:\right.$ $\left.k^{\times} \cap \prod_{p} N U_{\mathfrak{p}}\right)=(\varepsilon: \eta)$. Thus the theorem is proved. 


\section{REFERENCES}

[1] A. Fröhlich, The genus field and genus group in finite number field, Mathematika, 6 (1959), 40-46.

[1'] A. Fröhlich, The genus field and genus group in finite number field, II, Mathematika, 6 (1959), 142-146.

[2] H. Hasse, Zur Geschlechtertheorie in quadratischen Zahlkörpern, J. Math. Soc. Japan, 3 (1951), 45-51.

[3] S. Iyanaga and $T$. Tamagawa, Sur la theorie du corps de classes sur le corps de nombres rationelles, J. Math. Soc. Japan, 3 (1951), 220-227.

[4] K. Iwasawa, A note on class numbers of algebraic number fields, Abh. Math. Sem. Univ. Hamburg, 20 (1956), 257-258.

[5] S-N. Kuroda, Über die Klassenzahl eines relativ-zyklischen Zahlkőrpers vom Primzahlgrad, Proc. Japan Akad. 40 (1964), 623-626.

[6] H. Leopoldt, Zur Geschlechtertheorie in abelschen Zahlkörpern, Math. Nachr., 9 (1953), 351-362.

[7] A. Yokoyama, On class numbers of finite algebraic number fields, Tôhoku Math. J., 17 (1965), 349-357.

Mathematical Institute

Kanazawa University 